\title{
REESTRUCTURACIÓN DE LA ESCUELA DE POSTGRADO DE LA UNIVERSIDAD NACIONAL MAYOR DE SAN MARCOS
}

\author{
RESTRUCTURING POST-GRADE SCHOOL OF SAN MARCOS UNIVERSITY
}

\section{Raúl Arrarte Mera*}

Docente Asociado de la Facultad de Ciencias Contables, UNMSM

[Recepción: Enero de 2010 / Conformidad: Marzo de 2010]

\section{RESUMEN}

Este trabajo de investigación, financiado por el Vicerrectorado de Investigaciones de nuestra Universidad Nacional Mayor de San Marcos, correspondiente al año 2009, tiene por finalidad proponer a la autoridad competente, la reestructuración ordenada de la Escuela de Postgrado que orgánicamente cuenta, en la actualidad, con 20 Unidades dependientes administrativamente de cada Facultad, que de acuerdo al artículo $35^{\circ}$ del Estatuto vigente, "se encarga de establecer las políticas de altos estudios y de planificar, normar, supervisar y coordinar las Maestrías y Doctorados”. norma que rige desde 1984, bajo la Ley N.o 23733, conjuntamente con su Reglamento Interno. Como es de suponer, dichos instrumentos se hiceron en otro tiempo y en otro espacio, sin imaginar la actual competencia global, que viene remeciendo a las organizaciones sean estas lucrativas o de servicios, como es el caso de las universidades públicas.

Los resultados que se esperan obtener son: optimizar la capacidad instalada de las Unidades de Postgrado, incrementar los ingresos propios para la Universidad y, simultáneamente, contribuir a mejorar nuestra imagen

\begin{abstract}
This research, funded by the Research Vice-Rector of our Mayor de San Marcos University, since 2009, is it has to propose the competent authority, the orderly restructuring of the Graduate School, which has currently 20 administrative in each faculty, which, according to 35 article of the Statutes, "is responsible for establishing policies of higher learning and to plan, regulate, supervise and coordinate Masters and $\mathrm{PhD}$ 's, rule has been governing since 1984, under Law No. 23733, together with its internal regulation procedures. As you can imagine that instruments were made for other time and place, without imagine the current global competition, which is shattering these organizations, being these nonprofit or services ones, as is the case of public universities.

The results expecte to get are: the capacity of Postgraduate Units, increasing own revenue for the university, and simultaneously help to improve our institutional image contributing to the economic and social development, promoting the application of science and technology.
\end{abstract}

* Contador Público Colegiado, Doctor en Ciencias Contables y Empresariales y Magíster en Administración. Actual Director del Instituto de Instigaciones en Ciencias Financieras y Contables. E mail: raularrarte@speedy.com.pe 
institucional coadyuvando al desarrollo económico y social del país, promoviendo la aplicación de ciencia y tecnología.

Las unidades participantes serán, como muestra, las unidades de postgrado: Administrativas, Contables y Económicas.

Palabras clave: Escuelas de Postgrado, Unidades de Postgrado, Maestrías y Doctorados, Modernización de la Escuela de Postgrado, Actualización de la Escuela de Postgrado, Reestructuración de Unidades de Postgrado.

\section{INTRODUCCIÓN}

En el año 2004, el responsable del presente Proyecto se graduó de Doctor en Ciencias Contables y Empresariales en la Facultad de Ciencias Contables con la tesis denominada "La Cooperación - Universidad - Empresa - Estado en el Perú (Caso UNMSM, 1997-2002), donde se sostiene la necesidad de simplificar su organización para reducir costos, en base a la información financiera para hacerla más eficiente en la toma de decisiones, mejorar la calidad académica, solucionar sus problemas cotidianos que sin la función principal de coordinación no solo vertical, como se da en el presente, sino horizontal, resulta fundamental para competir en el mercado nacional e internacional.

Sin embargo, de esa fecha a esta parte, (del 2004 al 2009) nada ha cambiado en la Universidad. Nuestras autoridades no han podido construir el puente entre la universidad y la empresa para "realizar investigación en humanidades, ciencia y tecnología adecuándolas a los requerimientos nacionales con el objeto de ofrecer alternativas eficaces y viables para su propio desarrollo y la transformación socio-económica del país..." o "...vincular a la comunidad, por medio de mecanismos de interacción dinámica destinados a recoger la experiencia
The participating units will be, as shown, the postgraduate units: Administration, Accounting and Economics.

Keywords: Graduate Schools, Units Graduate, Masters and $\mathrm{PhD}$, Modernization of the Graduate School, Update of the Graduate School, Graduate Restructuring Unit.

y conocimiento que se producen fuera del claustro y a extender su acción y servicios hacia ella"; o "contribuir al estudio y enjuiciamiento de la problemática nacional e internacional, con plena independencia de criterio, planteando alternativas de solución..." como lo establecen los incisos $c$, e y $f$ del artículo $6^{\circ}$, del Reglamento de la Universidad, que en la práctica resultan, hasta ahora, letra muerta.

El proceso es muy lento y, pese a la creación del Vicerrectorado de Investigaciones, es fundamental la generación de ingresos propios, a la luz del Presupuesto del 2009, que acaba de aprobar el Parlamento Nacional, que no incrementará el ingreso por investigación y tecnología para las universidades públicas. Mensaje que nos obliga a mejorar los órganos, como las unidades de postgrado, en cuanto a incrementar sus ingresos para viabilizar los fines de nuestra alma máter.

\section{OBJETIVOS}

\section{Objetivo general}

Proponer a la Escuela de Postgrado flexibilizar el Reglamento sobre Otorgamiento de Grados y Títulos tanto en forma individual como por equipos de diferentes menciones (con un 
máximo de cinco miembros), como viene desarrollando el mercado internacional.

\section{Objetivos específicos}

1. Revisar los actuales planes de estudio con el objeto de normarlizarlos por sector para fusionar, potenciar o eliminar menciones, que eviten la competencia entre unidades de postgrado.

2. Normalizar las pensiones mensuales a nivel de la Universidad, estableciendo mínimos que permitan un ingreso adecuado para la Facultad pero también el pago de honorarios mínimos para nuestros docentes.

3. Definir políticas generales sobre duración de las menciones, en función del tiempo y en función de créditos.

4. Proponer el otorgamiento de grados y títulos tanto en forma individual como por equipos.

5. Facilitar y ampliar el uso y frecuencia de la capacidad instalada de las unidades de postgrado a nivel de la Universidad, sin tener en cuenta si es o no de tal o cual Facultad, sino que todos formamos parte de la misma alma máter. Así, la continuidad de los turnos podrían ser de Mañana, Tarde, Noche, solo sábados, solo domingos, con doble límite: el número de créditos aplicados y/o el tiempo de dos años, como ahora se vienen exigiendo.

6. Desarrollar normas de ética funcional para los directores del postgrado y el régimen de remuneraciones para incentivar el mayor uso de la capacidad instalada de las unidades a su cargo, buscando cambiar la mentalidad de Administrador a Gerente de Área.

\section{METODOLOGÍA DE INVESTIGACIÓN DEL ESTUDIO}

Esta investigación es de tipo aplicativa o tecnológica. El trabajo así desplegado, tiene el carácter de estudio descriptivo. Y como métodos de investigación, se ha utilizado la observación y el deductivo, que han permitido comparar y contrastar los resultados obtenidos.

\section{PRINCIPALES HALLAZGOS}

Las alternativas de solución a los problemas que afectan el desarrollo de la Escuela de Postgrado, se ubican en dos parámetros temporales: el largo y el mediano plazo.

1. En el largo plazo, formular un plan estratégico que contemple la construcción de un edificio fuera del Campus Universitario, integrado por comisiones de coordinación por sectores: Ciencias Básicas, Ciencias de la Salud, Ingenierías, Económico-Empresariales y Humanidades. Para analizar y desarrollar, el aspecto académico-económico-financiero de la Escuela de Postgrado. Para desarrollar un Proyecto de Inversión y facilitar su financiamiento, aprovechando el boom de construcción civil que vive el país.

2. En el mediano plazo, las soluciones que presentamos se analizan en detalle para tomar las medidas correctivas que sean necesarias para llevarlas a la práctica. La muestra utilizada en este estudio esta integrada por las Facultades del Sector Económico-Empresarial: Ciencias Administrativas, Ciencias Contables y Ciencias Económicas.

En el presente artículo, solo abordaremos los problemas de corto plazo, de fácil solución, si verdaderamente existe la actitud mental positiva para abordarlos. 
1. REVISIÓN DE PLANES DE ESTUDIOS DE LAS UNIDADES DE POSTGRADO DEL SECTOR ECONÓMICO-EMPRESARIAL

\section{Facultad de Ciencias Administrativas}

Según información proporcionada por esta Facultad a los candidatos, las diferentes menciones de maestría y doctorado, son las siguientes:

\section{Maestría}

1. Gestión Empresarial

2. Dirección de Recursos Humanos

3. Marketing

4. Finanzas y Valores

5. Comercio Internacional y

6. Finanzas Internacionales

7. Gestión Pública.

\section{Doctorado}

8. Ciencias Administrativas.

\section{Comentario}

Normalización. Sobre el nombre del curso de Metodología de Investigación

En las menciones desarrolladas por catedráticos nacionales: Gestión Empresarial, Dirección de Recursos Humanos, Marketing y Finanzas y Valores, el curso toma el nombre de Tesis II: Análisis Estadísticos y Tesis III: Desarrollo del Trabajo de Investigación. Todos tienen un crédito de 5.0.

Sin embargo, en la Mención de Gestión Pública, el curso se subdivide en: Seminario de Tesis I y Seminario de Tesis II, su crédito es de 4.0.

En el Doctorado, el curso cambia de nombre y se denomina: Investigación I, In- vestigación II, Investigación III, Investigación IV. Todos tienen un crédito de 6.0.

En las menciones desarrolladas por catedráticos franceses y nacionales: Comercio Internacional y Finanzas Internacionales, no aparece ningún curso de Metodología de Investigación.

\section{Facultad de Ciencias Contables}

Según información proporcionada por esta Facultad a los candidatos, las diferentes menciones de Maestría y Doctorado, son las siguientes:

\section{Maestría}

1. Auditoría en la Gestión Empresarial

2. Auditoría en la Gestión y Control Gubernamental

3. Banca y Finanzas

4. Comercio Internacional y Aduanas

5. Contabilidad de Gestión

6. Costos y Presupuestos en la Gestión Estratégica

7. Política y Sistema Tributario

8. Auditoría tributaria

\section{Doctorado}

9. Ciencias Contables y Empresariales

\section{Comentario}

\section{Normalización. Sobre el nombre del curso} de Metodología de Investigación

En las Menciones desarrolladas por Catedráticos Nacionales: Auditoría en la Gestión Empresarial, Auditoria en la Gestión y Control Gubernamental, Banca y Finanzas, Comercio Internacional y Aduanas, Contabili- 
dad de Gestión, Costos y Presupuestos en la Gestión Estratégica, Política y Sistema Tributario, Auditoría Tributaria, el curso toma el nombre de Seminario de Investigación Temática, Investigación Aplicada I, Investigación Aplicada II. Todos tienen un crédito de 8.0, 9.0 y 10.0 , respectivamente.

En el Doctorado, el curso cambia de nombre y se denomina: Tesis I, Tesis II, Tesis III. Todos tienen un crédito de 8.0, 10.0 y 12.0

\section{Facultad de Ciencias Económicas}

Según información proporcionada por esta Facultad a los candidatos, las diferentes menciones de maestría y doctorado, son las siguientes:

\section{Maestría}

1. Finanzas

2. Desarrollo Empresarial y Local

3. Gestión y Políticas Públicas

4. Economía y Gestión en la Educación

\section{Doctorado}

\section{Gestión Económica Global}

\section{Comentario}

\section{Normalización. Sobre el nombre del curso} de Metodología de Investigación

En las Menciones desarrolladas por Catedráticos Nacionales: Finanzas, Desarrollo Empresarial y Local, Gestión y Políticas Públicas, el curso toma el nombre de Métodos y Técnicas de Investigación, Seminario de Investigación I, Seminario de Investigación II, Seminario de Tesis I, Seminario de Tesis
II, Seminario de Tesis III. Todos tienen un crédito de 8.0, 9.0 y 10.0, respectivamente.

En el Doctorado, el curso cambia de nombre y se denomina: Metodología de la Investigación. Todos tienen un crédito de $8.0,10.0$ y 12.0 .

\section{Efectos que genera esta situación}

\section{Sobre normalización}

a) El curso emblemático "Metodología de la Investigación Científica”, toma distintos nombres según se trate de maestría o doctorado y de acuerdo a la Facultad donde se dicte.

b) Los créditos por este Curso son diferentes. En Ciencias Administrativas, el rango va de 4 a 6. En Ciencias Contables, en Maestría, va de 8 a 10 créditos. Mientras que en Doctorado, de 8 hasta 12 créditos. En Ciencias Económicas, la situación es similar a Ciencias Contables.

c) En cuanto a los créditos por semestre, nuevamente, no hay uniformidad. En Ciencias Administrativas y Contabilidad, son un total de 72. En Ciencias Económicas van de 78,79 a 80 créditos.

\section{Sobre fusión y eliminación de menciones}

$\mathrm{Al}$ respecto, existe un error de percepción. Se cree que una Facultad es más competitiva que otra cuanto más menciones ofrece al mercado. Lo que en realidad deseamos es captar la mayor cantidad de postulantes, porque aumentarán los ingresos, tanto para la Universidad como para los profesores a cargo de los cursos, mejorando la rentabilidad al eliminar costos innecesarios.

Por ejemplo, en Ciencias Administrativas, existen dos menciones con nombres 
similares: FINANZAS Y VALORES, a cargo de profesores nacionales; y FINANZAS INTERNACIONALES, con intervención de profesores extranjeros. El primero cuesta S/.900 mensuales; el último, S/. 1200. ¿Qué curso interesará al postulante? ¿El más barato o el más caro? La respuesta está en analizar la demanda. Si deseamos diferenciar y elevar el nivel de nuestras maestrías, se debería eliminar la mención de FINANZAS Y VALORES. Corrección que permitirá promocionar la demanda de FINANZAS INTERNACIONALES.

En Ciencias Contables sucede el mismo fenómeno. Dos menciones compiten entre sí: CONTABILIDAD DE GESTIÓN y COSTOS Y PRESUPUESTOS EN LA GESTIÓN ESTRATÉGICA. El resultado: se divide la participación de postulantes y se deja de aprovechar la única mención de costos que existe en el mercado nacional. Por tanto, en el caso bajo análisis, el Comité Directivo debe definir si elimina uno de ellos, fusionarlos o crear una nueva mención con nombre diferente.

\section{Sobre competencia entre facultades del sector}

Esta situación se presenta en las siguientes menciones que ofrece el sector económicoempresarial:

a) Ciencias Administrativas ofrece Finanzas y Valores.

b) Ciencias Contables presenta Banca y Finanzas.

c) Ciencias Económicas: Finanzas, a secas.

¿Qué decisión tomará el postulante? Si decide por el precio, la mención a) cuesta S/. 900; la mención b), S/.500; la mención c), S/. 800. Resulta que la más baja es la oferta de Ciencias Contables. La más alta es de
Ciencias Administrativas. La lógica nos lleva a pensar que elegirá el precio más bajo. En desmedro de las otras menciones. Ganará el postulante y la Facultad elegida, pero perderá la Universidad en su conjunto.

¿Qué hubiera ocurrido si las tres Menciones tuvieran el mismo precio? Obligará al postulante al evaluar la estructura física, el contenido del currículo, la cercanía a su lugar de trabajo, la calidad de los profesores, etc. ¿Quién gana y quién pierde? Ganará el postulante y la Universidad, porque se ha eliminado la competencia entre pares. Perderá la Facultad que no ha sabido diferenciar su mención.

\section{Cómo superar los inconvenientes}

La solución pasa por un proceso de COORDINACIÓN entre los equipos de las diferentes Unidades de Postgrado

a) Sobre normalización del curso "Metodología de la Investigación Científica". Reunir en una mesa de trabajo al Director de la Unidad de Postgrado y a su Coordinador Académico con sus pares del sector económico-empresarial a fin de definir la uniformidad del nombre a emplearse en el sector. Esta alternativa es la más conveniente para la Universidad porque proyecta la imagen de una gestión coordinada.

b) Sobre fusión y eliminación de menciones. Corresponderá al Director de Postgrado y su Comité Directivo, analizar en primera instancia la consecuencia de esta realidad. Si ambas menciones tienen la misma cantidad de postulantes, habrá que nivelar hacia arriba el valor de las mensualidades. Se elimina la brecha de competencia interna. Si el Comité Directivo prioriza la calidad de la mención, 
habrá que eliminar aquella que compite por precio. Similar enfoque debe ocurrir con las menciones que ofrecen el mismo producto pero con diferente nombre. $\mathrm{O}$ fusión por absorción o fusión con creación de una nueva mención. Rectificación que mejorará el nivel de ingresos de la Facultad, porque reducirá costos innecesarios.

c) Sobre competencia entre facultades. Aquí la decisión resulta un poco más delicada porque hay que COORDINAR Y COOPERAR mutuamente entre los comités directivos de todo el sector. Un tema que puede contribuir a orientar el nivel de discusión es el siguiente:

El proceso administrativo tiene cuatro etapas: PLANIFICAR, ORGANIZAR, DIRIGIR y CONTROLAR. La Economía tiene dos áreas claramente definidas: MICROECONOMÍA Y MACROECONOMÍA. En función de este análisis, los comités directivos del sector deben cooperar y coordinar en definir los espacios que les corresponde como ciencia, de acuerdo a nuestra apreciación: a) Corresponde a Ciencias Administrativas los procesos de ORGANIZAR Y DIRIGIR, específicamente el área de Gestión en Micro y Macroeconomía.

b) Corresponde a Ciencias Contables los procesos de PLANIFICAR Y CONTROLAR en Micro y Macroeconomía.

c) Corresponde a Ciencias Económicas todas las etapas del Proceso Administrativo pero en el área Macroeconómica.

Si los comités directivos coordinan en estos segmentos, recién la Universidad Nacional Mayor de San Marcos estará en capacidad de competir, como institución integrada, ante la universidad privada e internacional.

\section{PENSIONES MENSUALES QUE SE COBRA Y HORAS-HOMBRE QUE SE PAGA A LOS PROFESORES EN EL POSTGRADO}

\section{Pensiones mensuales que se cobra en el sector económico-empresarial}

Analicemos, en primer lugar, la variedad de cobros en el Postgrado:

\begin{tabular}{|l|c|c|c|c|}
\hline \multirow{2}{*}{ Postgrado } & \multicolumn{2}{|c|}{ Maestría } & \multicolumn{2}{c|}{ Doctorado } \\
\cline { 2 - 5 } & Matrícula & Pensión mes & Matrícula & Pensión mes \\
\hline & & & & \\
\hline Administrativas & $\mathrm{S} / .500$ & $\mathrm{~S} / .900$ & $\mathrm{~S} / .700$ & $\mathrm{~S} / .1300$ \\
\hline & $\mathrm{S} / .500$ & $\mathrm{~S} / .1200^{(1)}$ & & \\
\hline & & & & $\mathrm{S} / .900$ \\
\hline Contables & $\mathrm{S} / .500$ & $\mathrm{~S} / .500$ & $\mathrm{~S} / .700$ & \\
\hline & & & & $\mathrm{S} / .1100^{(1)}$ \\
\hline & & & $\mathrm{S} / .700$ & \\
\hline Económicas & $\mathrm{S} / .500$ & $\mathrm{~S} / .600$ & & \\
\hline & & $\mathrm{S} / .800$ & & \\
\hline
\end{tabular}

(1) Curso Internacional. 
Nota: La Escuela de Postgrado tiene tres categorías para efecto de matrículas y pensiones:

1. Docentes y personal administrativo de la UNMSM.

2. Docentes de universidades nacionales y egresados de la UNMSM.

3. Egresados de otras universidades.

Para el cuadro bajo análisis, hemos tomado las matrículas y pensiones de la tercera categoría.

\section{Comentario}

Se observa en las matrículas de las diferentes unidades de postgrado que hay uniformidad en el cobro: S/. 500 para las maestrías y S/.700 para el doctorado. No ocurre lo mismo con las pensiones mensuales. En nuestra opinión, el criterio también debe ser uniforme. El postulante elegirá la mención no porque cueste menos, sino por los colaterales de la oferta: el currículo, la infraestructura o la composición de sus profesores.

Para evitar la competencia entre pares, urge coordinar la uniformidad en las pensiones. El diferencial sería la presencia de profesores extranjeros con la oferta del doble grado académico simultáneo.

\subsection{Horas-hombre que se paga en el sec- tor económico-empresarial}

Analicemos los criterios que se manejan para el pago de la hora académica:

\begin{tabular}{|l|c|c|c|c|}
\hline \multirow{2}{*}{ Postgrado } & \multicolumn{2}{|c|}{ Magister } & \multicolumn{2}{c|}{ Doctor } \\
\cline { 2 - 5 } & Tiempo & Costo & Tiempo & Costo \\
\hline Administrativas & 40 minutos & $\mathrm{S} / .80$ & 40 minutos & $\mathrm{S} / .100$ \\
\hline Contables & 60 minutos & $\mathrm{S} / .100$ & 60 minutos & $\mathrm{S} / .110$ \\
\hline Económicas & 45 minutos & $\mathrm{S} / .100$ & 45 minutos & $\mathrm{S} / .100$ \\
\hline
\end{tabular}

\section{Comentario}

No existe una lógica razonable para discriminar el pago hora-hombre a los Profesores. La única diferenciación que debe darse en esta variable es por el Grado. Urge aquí, normar el tiempo y la hora-hombre para el Profesor que presta sus servicios en toda la Universidad Nacional Mayor de San Marcos, con una tasa uniforme. Es oportuno recordar que la hora-hombre actual generalizada es de S/. 100, rige desde el año 1993. Tasa que debe revisarse cada año al momento de desarrollar los presupuestos aplicando la técnica de costo-volumen-utilidad.

\section{DEFINIR MENCIONES EN FUNCIÓN DEL TIEMPO Y DE CRÉDITOS}

Entre los desafíos que ha creado la globalización en la capacitación de ejecutivos y promovido la competencia interuniversidades, está el uso óptimo del tiempo. Las clases se extienden a horarios de mañanas, tardes, noches y fines de semana. Ha desaparecido la "semana inglesa”, costumbre que establecía los descansos en sábados y domingo. "El tiempo vale más que el dinero", nos dice un slogan bancario nacional. Sin embargo, el Estatuto de la Universidad no contempla estas realidades. Y 
las unidades de postgrado, deben adaptarse o morir en el intento, ante la universidad privada que sí ofrece estas alternativas.

A continuación, artículos atingentes al tema bajo análisis, normados por el Estatuto de la Universidad:

“Artículo 312 ${ }^{\circ}$. Los cursos de Post-Grado del currículo de Segunda Especialidad, Maestrías o Doctorados, pueden convalidarse entre sí, siempre y cuando el contenido y los créditos sean equivalentes".

“Artículo $313^{\circ}$. Los currículo de las Maestrías comprenden una duración mínima de dos años o 4 semestres académicos. Los cursos avanzados, seminarios, investigación bibliográfica, investigación avanzada y entrenamiento en la docencia universitaria, tendrán un contenido mínimo total de 72 créditos".

“Artículo 315 . Los currículos del doctorado comprenden una duración mínima de dos años o cuatro semestres académicos. Los cursos avanzados, seminarios y desarrollo de la investigación original y crítica tendrán un contenido mínimo total de 72 créditos".

La conclusión de los artículos mencionados es: Dos años o cuatro semestres académicos para las maestrías y doctorados en términos de tiempo. Y de 72 créditos como contenido mínimo. No hay la alternativa de cubrir los 72 créditos en un periodo inferior a dos años, menos a un año.

En un mercado tan exigente como competitivo, la Universidad Nacional Mayor de San Marcos debe tener la flexibilidad de ofrecer las maestrías y doctorados tanto en un año de doce meses como en un periodo de 5 años. Lo que permitiría a los graduandos programar sus cursos en función de la disponibilidad de su tiempo y de su capacidad de pago. ALTERNATIVA QUE ESTÁ LEJOS DE SER ALCANZADA EN LOS MOMENTOS ACTUALES BAJO LA REGLAMENTACIÓN VIGENTE.

\section{OTORGAMIENTO DE GRADOS Y TÍTULOS EN FORMA INDIVIDUAL COMO POR EQUIPOS}

Dos grandes debilidades tiene la Universidad en camino hacia la globalización de sus actividades visto desde la gran competencia en un mercado abierto no frente a las universidades nacionales públicas o privadas, sino frente a las maestrías internacionales con oferta completa de financiamiento "estudie ahora y pague después", donde los estudios son a tiempo completo durante 12 meses y todos los egresados concluyen con sus grados correspondientes:

1. ¿Cómo incrementar el porcentaje de graduados que actualmente es mínima contribuyendo así a mejorar nuestra imagen institucional y coadyuvando al desarrollo económico y social del país?

2. ¿Cómo mejorar la cooperación interfacultades coadyuvando a integrar equipos con múltiples menciones para desarrollar trabajos de investigación multidisciplinarios promovidos por el Vicerrectorado de Investigación?

Las causas que podríamos identificar son muchas, pero las principales son las siguientes:

a) Los años 80 eran diferentes a los tiempos que vivimos en la actualidad. Nuestros legisladores no imaginaban siquiera que la Universidad debía servir a la empresa y al Estado. Hoy es común hablar de la cooperación Universidad-Empresa-Estado. $\mathrm{Su}$ estructura organizacional, repartida en 20 Facultades, no facilita la comunicación horizontal. Su organización académico-administrativa se sustenta en el régimen de Facultades. Pero se reconoce que para lograr la óptima satisfacción de sus necesidades orgánico-funcionales se orienta por los principios de integración, descentralización y desconcentración. 
b) La función de las unidades de postgrado era capacitar a sus docentes para orientarlos a la investigación. Hoy, los alumnos que asisten en un 95\%, son profesionales del sector público y privado que lo hacen con la finalidad de ser competitivos y actualizarse dada la velocidad de los cambios que genera el conocimiento científico.

c) El Estado, cada vez, se aleja del convencimiento de priorizar la educación pública, al no dar su apoyo decisivo a la reforma del sistema universitario nacional para el incremento de sus magros presupuestos hacia la mejora del nivel de vida de sus docentes. Porque la infraestructura es óptima gracias precisamente al incremento de sus ingresos autogenerador que sirven a tal fin, pero que, en adelante, se precisa de un trabajo fino de ingeniería económica para continuar aumentando los ingresos.

Veamos el caso de la Escuela de Negocios de Valparaíso de la Universidad Adolfo Ibáñez, para conocer cómo actúa la competencia en nuestra Región ${ }^{1}$ :

1. La Escuela de Negocios propone una lista de empresas y temas a desarrollar. Sin embargo, los estudiantes pueden también postular proyectos de su interés si cuentan con la aprobación de la empresa interesada.

2. A través de la participación de los estudiantes en estudios específicos y concursos, tales como el organizado por el World Resources Institute de Washington, USA, al cual se le solicita un Plan de Negocios de Empresas Sostenibles.

La tesis final es un proyecto de consultoría que se desarrolla en el segundo semestre del programa (se debe recordar que son es- tudiantes de tiempo completo por un periodo de 12 meses). En nuestro caso, podría ser en el cuarto semestre de la maestría.

Finalizado el estudio, los graduandos deben presentar el trabajo ante un Jurado integrado por cuatro (4) personas. Tres miembros de la Escuela y un directivo de la empresa consultada, mientras que los primeros califican con el 50\% de la nota y el ejecutivo empresarial, el otro 50\%.

En cuanto al Profesor Asesor, se excluye de ser parte del Jurado. Asiste a la reunión de presentación con la empresa, y luego va asesorando al grupo de trabajo en función a los avances de la tesis.

Para optimizar el apoyo de la empresa privada, la Escuela cuenta con un Consejo Consultivo integrado por importantes empresarios, de suerte que, proponen temas a la Universidad para que ella las tome directamente y así ahorrarse el diferencial de costos que implica pagar a una consultora nacional y/o extranjera 'para el análisis de un tema ya que tienen que hacer un abono de dinero a la Universidad y que sirve para financiar algunos gastos del grupo de trabajo en el desarrollo de la tesis.

El proyecto consiste en un trabajo aplicado, real (que actualmente viene exigiendo con justificadas razones el Vicerrectorado de Investigaciones de la Universidad), que responde a un requerimiento concreto de una Empresa o "Cliente", (palabra muy difícil de asimilar en San Marcos). Los alumnos trabajan directamente con la empresa solicitante, entrevistando a ejecutivos y recolectando la información necesaria para llevar a cabo el Proyecto. (Trasladamos el salón de clase al ambiente real de una Empresa). Los temas pueden ser individuales o por equipos máximo de cinco (5) alumnos, y siempre supervi-

1 Arrarte Mera, Raúl. (2004) “La Cooperación Universidad-Empresa-Estado en el Perú. (Caso UNMSM 1997-2002). Tesis de Doctorado. UNMSM. Lima - Perú. 
sados por un asesor nombrado por la Escuela. Así ganan todos: Universidad, Empresa y el país.

\subsection{Qué debemos realizar para llevar a la práctica las ideas planteadas}

1. Mejorar estructura organizacional de la Escuela de Postgrado buscando la inte- gración de todas las unidades de postgrado. En la integración por sectores es importante el intercambio de ideas del Director de la Unidad de Post grado, del Coordinador Académico con sus pares de las otras Facultades, situación que no esta ocurriendo en la actualidad. Como puede notarse, el problema es de $\mathrm{CO}$ ORDINACIÓN.

Para ello, proponemos el siguiente organigrama:

\section{DIRECCIÓN DE ESCUELAS DE POSTGRADO}

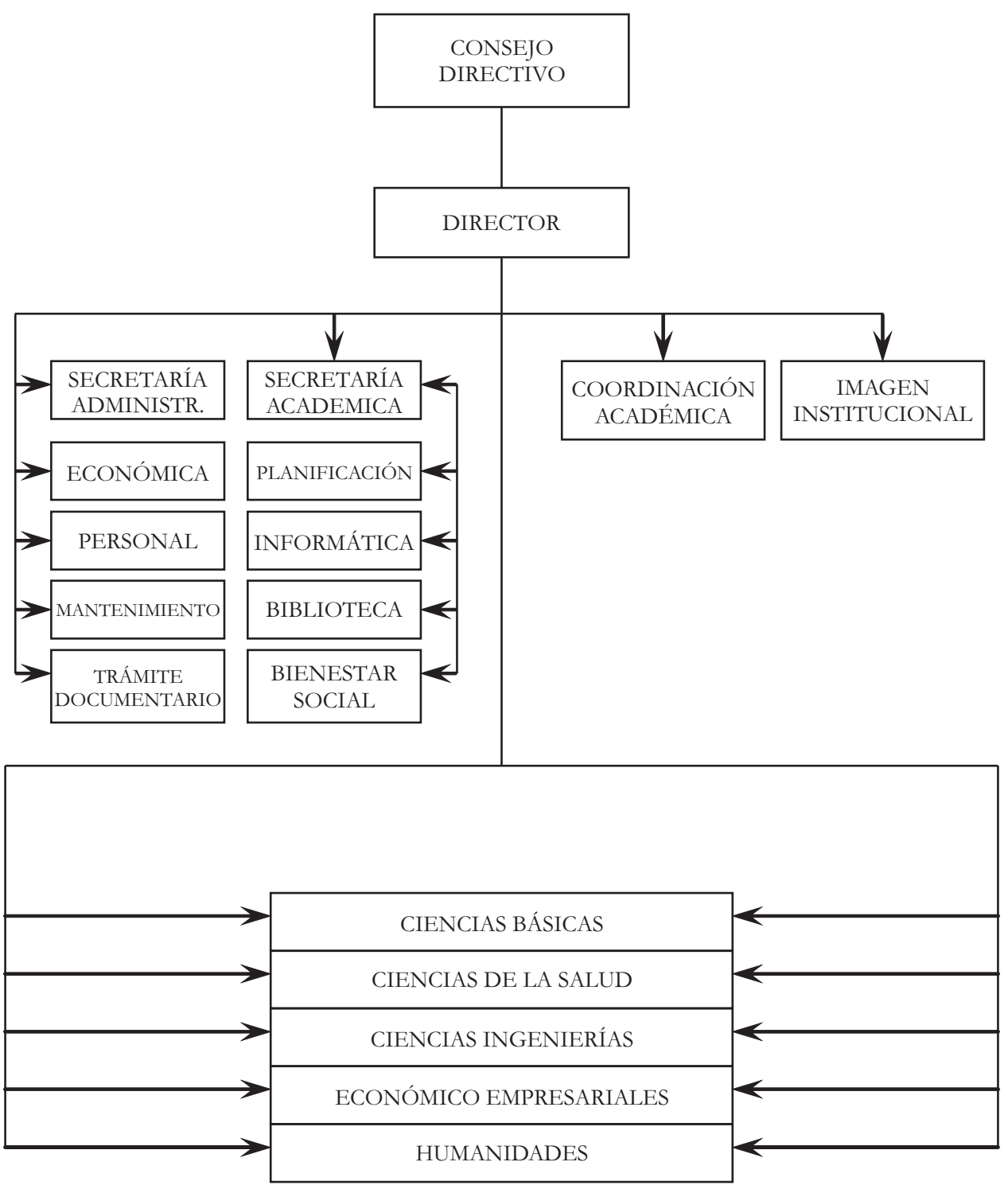


2. Modernizar el Reglamento de Grados y Títulos ingresando la figura de la graduación tanto en forma individual como por equipos. Integrar al Jurado un representante de la empresa privada y/o pública según el tipo de proyecto de investigación y todo aquello que contribuya a desarrollar el enfoque de Universidad-Empresa-Estado.

Se deben revisar los siguientes artículos del Estatuto de la Universidad Nacional Mayor de San Marcos:

“Artículo $\mathbf{1 0}^{\circ}$. La organización académico-administrativa de la Universidad $\mathrm{Na}$ cional Mayor de San Marcos se sustenta en el régimen de Facultades. Para lograr la óptima satisfacción de sus necesidades orgánico-funcionales se orienta por los principios de integración, descentralización y desconcentración.”.

"Artículo $11^{\circ}$. La Facultad es la unidad académico-administrativa básica que funciona como organismo descentralizado responsable de la formación académico-profesional, de perfeccionamiento, de investigación, de extensión universitaria y proyección social, de generación y promoción de cultura en aéreas afines del conocimiento.".

"Artículo $1^{\circ}$. Las Facultades gozan de autonomía de gobierno académico, económico y administrativa en el desarrollo de sus actividades, dentro de la planificación general y de las disposiciones de los órganos de gobierno de la Universidad, así como de las propias normas y reglamentos que rigen su organización y funcionamiento.".

"Artículo 780. Son atribuciones de la Asamblea Universitaria:

a) Modificar el Estatuto de la Universidad, requiriendo para ello, la mayoría absoluta de sus miembros hábiles. h) Acordar la creación, fusión y supresión de las Escuelas Académico-Profesionales, Institutos y Centros de Investigación, Unidades de Post-Grado y cualesquiera otros organismos de la Universidad siendo necesario para ello la aprobación por mayoría absoluta de sus miembros hábiles a proposición del Consejo Universitario. Acordar la creación, fusión, supresión de Facultades, requiriendo para ello, los dos tercios de los votos de sus miembros hábiles a propuesta del Consejo Universitario o por iniciativa de la propia Asamblea.

j) Disponer la reorganización de la Universidad y/o Facultades, requiriendo para ello los dos tercios de los miembros hábiles de la Asamblea.

Artículo 84'. Son funciones del Consejo Universitario:

d) Proponer a la Asamblea Universitaria la creación, fusión, supresión o reorganización de las Facultades, Escuelas Académico-Profesionales, Unidades de Postgrado, Departamentos Académicos, Institutos y Centros de Institutos y Centros de Investigación.

h) Ratificar el nombramiento, contratación, promoción y confirmar la no ratificación de los profesores de la Universidad, a propuesta de las Facultades.

j) Constituirse en instancia de apelación en las sanciones a los docentes, estudiantes, y personal administrativo y de servicio.

“Artículo 85. El Consejo Universitario funciona como plenario y por comisiones permanentes. Para su instalación y funcionamiento se requiere que el quórum sea la mitad más uno de sus miembros 
hábiles. Cuando se trate de asuntos de carácter administrativo podrá solicitar la presencia de los funcionarios correspondientes en calidad de asesores.

Todos los acuerdos del Consejo Universitario se toman en sesión plenaria.

Asimismo, los siguientes artículos del Nuevo Reglamento de Estudios y Evaluación de Maestrías y Doctorados:

Artículo $1^{\circ}$. La Unidad de Postgrado, es la unidad académica de la Facultad encargada de planificar, organizar y dirigir los estudios de Segunda Especialización y de organizar y dirigir los estudios de Maestría y Doctorado en el ámbito de su competencia.

Está a cargo de un Director, Profesor Principal o Asociado a dedicación exclusiva o tiempo completo, con grado de Doctor, y de un Comité Directivo integrado por dos tercios de profesores con grado de Doctor o Maestro y un tercio de alumnos de Post-Grado, designado por el Consejo de Facultad.

Artículo $310^{\circ}$. Los estudios de Maestría y Doctorado son normados, coordinados y supervisados por la Escuela de Post-Grado y dirigidos y ejecutados por la Unidad de Post-Grado de la Facultad por medio de sus profesores, con grado de Maestro y Doctor, en los ambientes respectivos. En casos excepcionales, se podrá celebrar convenios para realizar la enseñanza de las Maestrías y Doctorados fuera de los ámbitos de la Universidad

Artículo $311^{\circ}$. La creación de las Segundas Especialidades, las Maestrías y el Doctorado debe ser aprobada por el Consejo Universitario y ratificada por la Asamblea Universitaria a propuesta de la Unidad de Post-Grado de la Facultad y previo informe obligatorio de la Escuela de Post-Grado de la Universidad. En la propuesta se debe especificar el cumplimiento de los requisitos básicos que señale el Reglamento respectivo

Artículo $312^{\circ}$. Los cursos de Post-Grado de los currículos de Segunda Especialidad, Maestrías o Doctorados, pueden convalidarse entre sí, siempre y cuando el contenido y los créditos sean equivalentes.

Artículo $313^{\circ}$. Los currículo de las Maestrías comprenden una duración mínima de dos años o 4 semestres académicos. Los cursos avanzados, seminarios, investigación bibliográfica, investigación avanzada y entrenamiento en la docencia universitaria, tendrán un contenido mínimo total de 72 créditos.

Artículo $315^{\circ}$. Los currículos del Doctorado comprenden una duración mínima de dos años o cuatro semestres académicos. Los cursos avanzados, seminarios y desarrollo de la investigación original y crítica tendrán un contenido mínimo total de 72 créditos.

La pregunta que debemos contestar en el cortísimo plazo es, por qué vamos atrasados en el tiempo: ¿Habrá la entereza de una corriente de opinión favorable al interior del Consejo de Facultad, del Consejo Universitario y Asamblea Universitaria, para alcanzar esta meta que allanarían los problemas de la Universidad más antigua de América? Por que solo así lograremos incrementar el número de graduando, desarrollar proyectos multidisciplinarios y hablar a viva voz que vamos camino al desarrollo de la INVESTIGACIÓN, INNOVACIÓN y TECNOLOGÍA, en un ambiente de integración entre UNIVERSIDAD-EMPRESA-ESTADO. 


\section{SUB-EMPLEO DE CAPACIDAD INSTALADA EN LA ESCUELA DE POSTGRADO}

En toda Empresa, cualquiera sea su tamaño o sector de actividad, que brinde servicios, como el tema que nos ocupa, se presenta tarde o temprano, "y últimamente más temprano que tarde, la necesidad de bajar costes y ser más competitivo a la vez"2. Este autor nos dice que el consumidor es más exigente; el valor añadido, que con frecuencia tanto cuesta a las empresas conseguir en un mercado crecientemente competitivo, deja de ser tal muy rápidamente para pasar a ser simplemente algo esperado por el consumidor. Los mercados se internacionalizan de forma exponencial, ya no hay cotos cerrados de negocios, todos pueden llegar a todos lados y competir de igual a igual. En fin, todo confluye a una aparente paradoja: la necesidad de brindar al consumidor más calidad a menor coste. Sin embargo, esta paradoja es aparente, sobran los ejemplos de empresas que han podido solucionarla rompiendo algunos moldes, con ingenio y sobre todo con un esfuerzo y método de trabajo continuo y planificado

El cuadro arriba descrito, es real, está ocurriendo en el mercado global. Pero, como dice un viejo adagio: "Dios es Peruano". San Marcos, pese a sus contradicciones, contra lo que otros pudieran decir, "aún es un coto cerrado de negocios", pero no para las mayorías, la comunidad, los egresados, los gobiernos locales, regionales y nacionales, clientes naturales, a quienes deberíamos estar sirviendo mejor y con calidad, como Universidad Pública. Sí lo es para el status quo, que no permite los cambios de orienta- ción y que tiende a matar, lentamente, a la gallina de los huevos de oro. Pero que sí hay una salida: CON MÉTODO DE TRABAJO CONTINUO Y PLANIFICADO.

Para entender el problema, identifiquemos sus causas:

- Sin integración a un sistema gerencial que permita conocer oportunamente, los ingresos, gastos y resultados por maestrías dictadas. Sólo hay preocupación cuando hay ausencia de liquidez en la Facultad.

- Uso de locales en Unidades de Postgrado solamente de lunes a sábados en turnos nocturnos de 6 a 10 p.m. Libre de 8 a.m. a 6 p.m. Inclusive todo el domingo.

- Escasa cooperación Universidad-Empresa-Estado, como por ejemplo: Ausencia de proyectos de capacitación, para Empresas, a medida de sus necesidades.

- Cambios continuos en Dirección y Administración del Postgrado lo que genera inestabilidad de Docentes.

- Maltrato al profesor al abonar honorarios con retraso, lo que afecta la imagen institucional.

- Exceso en número de maestrías y escaso número de alumnos.

\subsection{Alternativas de solución}

1. Es preciso dar estabilidad a la Unidad, "buscando al hombre para el puesto, y no el puesto para el hombre". Cuando lo político se impone a lo técnico, los resultados resultan contraproducentes para todos. Necesitamos Gerentes que actúen como promotores y no administradores de presupuesto.

2 Gonzáles de las Cuevas, Luís Roldán. “10 Pasos para Aumentar su Rentabilidad”. Editorial Díaz de Santos. S.A. Prólogo Página XIII. Año 2006. Madrid, Buenos Aires, México. 
2. En las organizaciones modernas, existen dos columnas vertebrales: el cliente interno, que en este caso es el Profesor a cargo del Curso, y el cliente externo, el consumidor que es el alumno. Debe haber coordinación permanente para cancelar a fin de mes, los honorarios del Catedrático. Única manera de involucrarlo al proceso de aprendizaje para mejorar el clima organizacional.

3. Promover la INTEGRACIÓN a nivel de la Escuela de Postgrado, el uso intensivo de los espacios que disponen las Facultades y que no se emplean. Sean estos por las mañana, por las tardes o fines de semana de sábado y domingo. A cambio de un pago por alquiler que debería normarse al interior de la Universidad. Por ejemplo, la Unidad de Postgrado dispone de 10 Aulas que están libres de lunes a domingo de 8:00 a 18:00 horas. ¿Hay alguna Unidad en la Universidad que necesita espacio para el dictado de sus clases en otra Maestría? Este tipo de coordinación no se está desarrollando en la actualidad.

4. Un sector que el Postgrado no ha desarrollado en forma general, es su colaboración en la capacitación a los cuadros directivos de las regiones como es el caso de oferta de maestrías a medida de las necesidades de estas instituciones. Se pueden ofertar maestrías o diplomados en los siguientes turnos:

a) Mañanas: Lunes a viernes de 8:00 a 12:00 horas.

b) Tardes: Lunes a viernes de 14:00 a 18:00 horas.

c) Fines de Semana: Domingos de 8:00 a 12:00 horas.

¿Qué necesitamos para llevarlo a la práctica? Planificar y vender el producto a las entidades del sector público $\mathrm{y} / \mathrm{o}$ privado.
¿Se precisan vendedores que oferten los productos? Se puede preparar estudiantes del Pregrado que perfectamente pueden dedicarse a visitar a las entidades que pudieran interesarse o equipos de profesionales que recorran las diferentes instituciones del país. Es decir, promover, promocionar nuestros productos.

\subsection{Nuevo régimen de remuneraciones para los directores de las unidades del postgrado}

Un tema que va junto a lo anteriormente expresado, es la remuneración que se paga a los Directores de las Unidades de Postgrado. En actualidad es de S/. 3200 mensuales. Su financiamiento está dividido en dos partes: $70 \%$ a cargo de la Facultad y que se cumple puntualmente, como debe ser todo servicio que se recibe. $\mathrm{Y}$ el $30 \%$ a cargo de la Administración Central, que se paga tarde, mal y nunca. En nuestra opinión, para incentivar la participación activa de los Directores, creemos con fundadas razones, que el sistema de pago debe ser en función de resultados. Para que se dediquen exclusivamente a planificar el futuro. Que es la función actual de un ejecutivo. Creemos que podría fijarse en S/. 1000 fijo mensual y reconocerle un 10\% como sueldo variable, en función de los futuros ingresos que genera. Así todos participan en la generación de los ingresos: El Director, los Profesores, la Facultad y la Universidad en su conjunto.

Veamos lo que dice el Reglamento General de los Centros de Producción de la UNMSM, que podría darnos una idea de cómo se reconoce el trabajo de los Gerentes de estas Unidades:

Artículo $30^{\circ}$. Las remuneraciones del personal contratado serán propuestas por el 
Gerente, aprobadas por el Directorio y ratificadas por el COGESA o Consejo Universitario, respectivamente

Artículo $32^{\circ}$. Concluido el ejercicio anual, los resultados del mismo en caso generen utilidades serán distribuidos en la siguiente proporción:

a) $60 \%$ para la Facultad como recurso propio y mejoramiento del respectivo CENPRO.

b) $20 \%$ para la Administración Central.

c) $20 \%$ como distribución de utilidades para los gestores y personal del CENPRO.

En el caso de los CENPRO de la Administración Central, se considerará el $80 \%$ como recurso propio.

Artículo $33^{\circ}$. La distribución del 20\% de utilidades tendrá la siguiente proporción:

a) $40 \%$ al Gerente o Director Gerente.

b) $20 \%$ entre los miembros del Directorio.

c) $40 \%$ entre el personal contratado y permanente del CENPRO.

En el caso de que el Gerente tenga también el cargo de Director la asignación que percibe sería solo el que corresponde al Gerente.

En definitiva el mensaje que deseamos transmitir en este trabajo de investigación, es pagar mejor a quien se lo merece, para que se dedique con alma, vida y corazón, a generar ingresos para la Unidad de Postgrado a su cargo. Las ideas están planteadas, solo falta la decisión política.

\subsection{Descentralización de la enseñanza a nivel nacional e internacional}

Otro tema colateral a lo anteriormente expuesto, es el desarrollo de las maestrías al interior del país y hasta el exterior. En visitas al interior del país: En el Norte, el Centro y el Sur, hemos podido observar cómo actúa CENTRUM de la Pontificia Universidad
Católica del Perú y ESAN, ahora convertida en Universidad Nacional. Viaja un Profesory una Secretaria con un equipo móvil compuesto por una Computadora Personal, un Ecran y una Multimedia. Alquilan un ambiente en el mejor hotel de la zona y allí dictan sus clases los días sábados y domingos. Un viaje por avión, ahora a la fecha, a cualquier parte del país es de una hora, aproximadamente. Por tanto, con la planificación correspondiente, se viaje el sábado a primera hora y se está de retorno el domingo a las 7 p.m.

La función que corresponde a la Escuela de Postgrado, es coordinar por ejemplo, que se movilicen por sectores, a nivel nacional ofreciendo servicios de maestrías y doctorados. La Universidad privada está posicionándose en Provincias. Es hora que San Marcos, actuando con mentalidad empresaria salga a competir de igual a igual con la universidad privada y extranjera.

\section{NORMAS DE ÉTICA FUNCIONAL PARA LOS DIRECTORES}

Hemos dejado para el final el tema de la ética, la Universidad más antigua de América no puede dejar de tener un Código de Ética cuya función primordial es la de promover y preservar nuestra Cultura Institucional basada en el respeto a los siguientes principios:

IMAGEN: Todo el personal, deberá estar comprometido con la Institución; les corresponde como responsabilidad conjunta, la promoción y preservación de su imagen positiva como un valor que pertenece a todos, que es compartido y del cual se es responsable por el solo hecho de compartir un ideal común y ser un miembro de la UNMSM.

PROBIDAD: El personal dela UNMSM, deberá actuar con rectitud, honradez, y honestidad, procurando satisfacer los intereses 
legítimos de la corporación, sus usuarios y la sociedad en su conjunto, y desechando el provecho o ventaja personal obtenido por sí o interpósitas personas. Profesar y practicar un claro rechazo a la corrupción en todos los ámbitos de desempeño de la UNIVERSIDAD y cumplir cabalmente con las normas vigentes.

Eficiencia: El personal de la UNMSM brindará calidad en cada una de las labores a su cargo, buscando el resultado más adecuado y oportuno.

Idoneidad: El personal de la UNMSM se desenvolverá con aptitud técnica, legal y moral en el desempeño de su labor. Propenderá a una formación sólida acorde a la realidad, capacitándose permanentemente para el debido cumplimiento de sus labores.

Veracidad: El personal de UNMSM se expresará con autenticidad en las relaciones laborales con todos los miembros de la corporación y con terceros.

Lealtad y obediencia: El personal de la Institución actuará con fidelidad y solidaridad hacia toda la UNMSM, cumpliendo órdenes que le imparta el superior jerárquico competente, en la medida que reúnan las formalidades del caso y tengan por objeto la realización de actos de servicio que se vinculen con las labores a su cargo, salvo los supuestos de arbitrariedad o ilegalidad manifiestas, los cuales deberá poner en conocimiento de la DIRECCIÓN correspondiente. Asimismo, actuará con reserva y diligencia en el manejo de la información que conoce,

Justicia y equidad: El personal de UNMSM actuará con permanente disposición para el cumplimiento de sus funciones, otorgando a cada uno lo que le es debido, actuando con equidad en sus relaciones con sus superiores, los subordinados y con la ciudadanía en general.

No es intención de este trabajo, presentar un borrador de un código de ética, pero si hacer notar que es indispensable en los tiempos que corren, complementar estas detalles para alcanzar los fines que perseguimos.

Finalmente, debemos considerar los siguientes temas:

a) Deberes de conducta ética del personal que trabaja en la UNMSM.

b) Prohibiciones de conducta ética al personal.

c) Incentivos y sanciones,

d) Disposiciones complementarias.

e) Funciones del tribunal de ética.

\section{CONCLUSIONES}

1. Escuela de Postgrado de la Universidad Nacional Mayor de San Marcos, a decir de su Prospecto de Admisión 2009- $\mathrm{I}^{3}$, "la Escuela de Postgrado, como parte de la nueva estructura académico-administrativa de San Marcos, desde su fundación está dedicada a la formulación y a la regulación de programas de estudio de postgrado. Estos programas abordan los diversos aspectos académicos más relevantes en virtud de la compleja realidad nacional y sin perder de vista la dinámica de los conocimientos del mundo globalizado". Ofrece 118 programas de maestría y 26 programas de doctorado. Pero, para aumentar su rentabilidad, reduciendo costos y ser más competitivo en el mercado, que es cada vez más complicado, necesita de la COORDINACIÓN Y LA COOPERACIÓN de las diferentes Direcciones integrado por Sectores, para

3 Maestrías y Doctorados. Prospecto de Admisión 2009.I. CEPREDIM. Página 05. Diciembre de 2008. Lima, Perú. 
normalizar el nombre de los Cursos, fusionar y eliminar menciones y delimitar los campos privativos de cada profesión, para evitar la competencia entre pares.

2. A la luz de la internacionalización de los mercados y la globalización de la economía, urge que la Escuela de Postgrado, implante políticas generales a nivel de toda la Universidad, en temas como las pensiones a cargo de los maestrandos, que se incrementen en función del mercado y contribuya a estabilizar los precios de la universidad privada. Así como también a definir las horas-hombre mínimas que se debe pagar al catedrático, y la normalización de la hora académica. Pensando además de mejorar los ingresos de la Universidad, en el bienestar del profesor, que tiene honorarios congelados desde 1993, y diferenciando el Grado de Magíster y Doctor.

3. La velocidad que imprime la vida moderna hace que la Universidad se adapte a las necesidades del cliente externo: el maestrando. Todo lo que contribuyamos hacer en su beneficio, pondrá a la Escuela de Postgrado, en igualdad de condiciones para ser más competitivo. En este sentido, es de necesidad pública, modernizar el Estatuto de la Escuela de Postgrado introduciendo mejoras en las Menciones tanto en tiempo (UN AÑO, DOS AÑOS, TRES AÑOS) y EN FUNCIÓN DE LOS CRÉDITOS. Es decir, UN AÑO, para estudios a tiempo completo. Dos Años para estudios a Medio Tiempo. Tres años para estudios solo fines de semana. De igual manera dejar en libertad del maestrando estudiar en función de su disponibilidad de tiempo aplicando el control del número de créditos necesarios para graduarse.
4. Para superar la insuficiencia de graduados que actualmente no superan el $5 \%$ de los egresados, proponemos la modernización del Reglamento de Grados y Títulos que permita la graduación tanto individual o por equipos multidisciplinarios con un máximo de cinco (5) alumnos por tesis. Lo que contribuirá a promover la investigación científica con equipos multidisciplinarios.

5. Es responsabilidad de la Escuela de Postgrado: PLANIFICAR, ORGANIZAR, DIRIGIR y CONTROLAR las actividades de las Unidades de Postgrado a su cargo. En tal sentido, se debe optimizar el continuo y permanente uso de la capacidad ociosa que pudieran tener algunas facultades en el perímetro de la ciudad Universitaria, pensando unitariamente como una Universidad y no como 20 Facultades independientes que compiten entre sí. Para alcanzar estos fines, se propone modificar la remuneración de los Directores de las diferentes Unidades de Postgrado, reconociendo sus ingresos en función de resultados. S/. 1000, como monto fijo, y una comisión mensual del $10 \%$ sobre el incremento de los ingresos generados, y cuyo pago se cumpla ordenadamente a fin de mes. Para hacer que dichas autoridades se identifiquen con los colores de la Universidad Nacional Mayor de San Marcos. Asimismo, la proyección al mercado interno y externo, debe ser coordinada por sectores, y no en forma independiente como ahora se viene haciendo.

6. Finalmente, es conveniente promulgar un Código de Ética que contribuya a preservar nuestra cultura institucional, a mantener y elevar el clima organizacional de la Universidad Nacional Mayor de San Marcos. Para hacer que todos y 
cada uno de sus miembros, nos sintamos orgullosos de pertenecer a la universidad más antigua de América.

\section{RECOMENDACIONES}

Para hacer realidad las conclusiones que se proponen en este trabajo de investigación, financiado por el Vicerrectorado de Investigaciones de nuestra Universidad se proponen las siguientes actividades:

1. Convocar a comisiones de trabajo al Director de la Unidad y su Coordinador Académico por Sectores:

a) Ciencias Básicas: Integrado por las Facultades de Ciencias Biológicas, Ciencias Físicas y Ciencias Matemáticas.

b) Ciencias de la Salud: Integrado por las Facultades de Farmacia y Bioquímica, Medicina Humana, Medicina Veterinaria, Odontología y Psicología.

c) Ingenierías: Integrado por las $\mathrm{Fa}$ cultades de Ingeniería Electrónica y Eléctrica, Ingeniería Geológica, Minera, Metalúrgica y Geográfica; Ingeniería Industrial, Ingeniería de Sistemas, Química e Ingeniería Química.

d) Económico - Empresariales: Integrado por las Facultades de Ciencias Administrativas, Ciencias Contables y Ciencias Económicas.

e) Humanidades: Integrado por las $\mathrm{Fa}$ cultades de Ciencias Sociales, Derecho y Ciencias Políticas, Educación. Para normalizar el nombre de los Cursos, fusionar y eliminar menciones y delimitar los campos privativos de cada profesión, para evitar la competencia entre pares.

2. Nombrar una comisión al interior de la Escuela de Postgrado, en presencia de todos los Directores de las Unidades de Postgrado, un grupo no mayor de cinco personas, para preparar un borrador de las políticas generales para normar, a nivel de la Universidad Nacional Mayor de San Marcos:

- Las pensiones mensuales por sector a cobrar.

- El valor de las horas - hombre a pagar por sectores.

- Definir un tiempo uniforme de las horas - hombre.

- Los que seguirán los procedimientos internos para convertirse en una Resolución Rectoral.

3. Nombrar una comisión al interior de la Escuela de Postgrado, en presencia de todos los Directores de las Unidades de Postgrado, un grupo no mayor de cinco personas, para preparar un Borrador de Modificación del Estatuto de la Escuela de Postgrado, para normar, a nivel de la UNMSM:

Introduciendo mejoras en las Menciones tanto en tiempo, (UN AÑO, DOS AÑOS, TRES AÑOS) y EN FUNCIÓN DE LOS CRÉDITOS. Es decir, un año, para estudios a tiempo completo. Dos años para estudios a medio tiempo. Tres años para estudios solo fines de semana. De igual manera dejar en libertad del Maestrando estudiar en función de su disponibilidad de tiempo aplicando el control del número de créditos necesarios para graduarse. Posteriormente, se seguirán los procedimientos internos para convertirse en una Resolución Rectoral.

4. Convocar a los Directores de las Unidades de Postgrado a un Forum para discutir la introducción en el Reglamento de Grados y Títulos la Cooperación Universidad-Empresa-Estado que permita la 
graduación tanto individual o por equipos multidisciplinarios con un máximo de cinco (5) alumnos por tesis. Lo que contribuirá a promover la Investigación científica con equipos multidisciplinarios. Que será la presentación de Proyectos de Consultoría al Sector Público y/o Privado con la presencia en el Jurado Calificador de un ejecutivo de la empresa bajo análisis.

5. Convocar a los Directores de las Unidades de Postgrado a una discusión técnica sobre el pago de remuneraciones a los Directores en función de resultados. Para incentivar el incremento de ingresos que sea de mutuo beneficio para todos los integrantes de la Universidad. A más rendimiento, más ingresos.

6. Nombrar una comisión al interior de la Escuela de Postgrado, en presencia de todos los Directores de las Unidades de Postgrado, un grupo no mayor de cinco personas, para preparar un borrador del Código de Ética que normará en el futuro las actividades de todos los funcionarios de la Universidad. Posteriormente se seguirán los procedimientos internos para convertirse en una resolución rectoral, de uso obligatorio a partir de su vigencia.

\section{REFERENCIAS BIBLIOGRÁFICAS}

1. Arrarte Mera, Raúl (2004). "La Cooperación -Universidad - Empresa - Estado- en el Perú”. (Caso UNMSM $1997-$ 2002). Lima, Perú. Tesis de Doctorado. UNMSM.

2. Consejo Nacional de Educación (2007). "Proyecto Educativo Nacional: Las Cifras".

202/ QUIPURAMAYoc | Vol. 17(33) 2010
3. Corporación Peruana de Aviación Civil S. A. (CORPAC) (2006). "Código de Ética y Conducta”. Lima, Perú.

4. Comisión Nacional para la Segunda Reforma Universitaria. "Diagnóstico de la Universidad Peruana: Razones para una nueva reforma universitaria. Año 2002".

5. Escuela de Postgrado. "Reglamento General de Estudios de Postgrado". Resolución Rectoral N. ${ }^{0}$ 02528-R-07. UNMSM. Lima, Perú.

6. Grupo de Promotores de Bolonia en España. (2005) "Preguntas Frecuentes sobre: La Estructura de las Enseñanzas Universitarias, el Grado y el Postgrado”. España.

7. Guerra-García, Roger (2006). "50 Años de las Universidades Peruanas.” Academia Nacional de Medicina. Anales 2006. Lima, Perú. p. 108.

8. Instituto de Investigaciones de la Amazonía Peruana. (2005) "Manual de Políticas de Gestión Institucional”. Iquitos, Perú.

9. Oficina de Coordinación de Centros de Producción. Reglamento General de los Centros de Producción. UNMSM.

10. Oficina General de Planificación. "Plan Estratégico Institucional. 2007-2011”. UNMSM.

11. Piovano, Mirta. (2006). "Características y Tendencias en la Formación del Postgrado de las Enfermeras". Argentina.

12. Ugarte Silva, Alfonso. "Plan Estratégico de la Unidad de Postgrado 2007-2009”.

13. Ugarte Silva, Alfonso. Facultad de Ciencias Contables. "Plan Estratégico 20082012”.

14. Villorria, Manuel (2009). "La Modernización del Estado, Hoy. Democratizando Instituciones". En revista: Nueva Gerencia Pública. Noviembre de 2009. Lima, Perú. 